\title{
Análisis de resultados métricos de una nube de puntos y una medición directa en el patrimonio edificado. El Santuario de La Montaña en Cáceres
}

\author{
Metric results analysis of point cloud and direct measure in building \\ heritage. Sanctuary of "La Montaña" in Cáceres \\ $\underline{\text { F. Serrano }}^{(*)}$, J. Saumell ${ }^{(*)}$, F. Berenguer ${ }^{(*)}$
}

\section{RESUMEN}

El objetivo del presente artículo consiste en realizar un estudio comparativo entre dos procedimientos utilizados en el levantamiento de un edificio: método directo sin instrumentación y escaneado láser que proporciona nubes de puntos de gran densidad. Se pretende analizar el grado de exactitud de los resultados y los recursos y medios técnicos precisos para llevar a cabo cada una de las tecnologías. La metodología consistirá en comparar dos trabajos relativos al mismo edificio. Por un lado, se cuenta con unos planos de planta y sección de un proyecto orientado a la rehabilitación del edificio. Por otra parte, se utiliza un escaneado con tecnología láser, del que se tomará una selección de puntos precisa para obtener la información necesaria para poder realizar los planos de planta y sección, equivalentes a los anteriores. El resultado consistirá en tener una información del grado de exactitud de cada método, valorando el esfuerzo técnico que requiere cada uno de ellos.

Palabras clave: Métodos de levantamiento arquitectónico; láser escaner 3D; nube de puntos.

\section{ABSTRACT}

This article makes a comparative study of two types of methods used in building construction. On one hand, the creation of drawings uses direct method, basically by measuring and sketching without instruments and, on the other hand, the creation of these plans by modern laser scanning. The aim is to analyze the accuracy of final results and to consider the technical and other resources needed to carry out each method. Two different works, concerning the same building are compared. More specifically works consist of plan views and section views from a project executed and a scanning with laser technology performed of some parts of the same building. From there we will take out the appropriate cross-section slices to get the information needed to make plan and section views. The outcome will be to get information on the level of accuracy and to assess the technical effort required in each case.

Keywords: Survey methods; 3D laser scanner; point clouds.

(*) Escuela Politécnica - Universidad de Extremadura, Cáceres (España)

Persona de contacto/Corresponding author: fsecan@gmail.com (F. Serrano)

Cómo citar este artículo/Citation: Serrano, F., Saumell, J., Berenguer, F. (2014). Análisis de resultados métricos de una nube de puntos y una medición directa en el patrimonio edificado. El Santuario de La Montaña en Cáceres. Informes de la Construcción, 66(534): e016, doi: http://dx.doi.org/10.3989/ic.12.059.

Licencia/License: Salvo indicación contraria, todos los contenidos de la edición electrónica de Informes de la Construcción se distribuyen bajo una licencia de uso y distribución Creative Commons Reconocimiento no Comercial 3.o. España (cc-by-nc). 


\section{INTRODUCCIÓN}

\subsection{Antecedentes}

El levantamiento gráfico de edificios existentes se ha estado realizando desde los inicios de la actividad arquitectónica, con especial relevancia en el estudio de edificios catalogados desde el punto de vista patrimonial, habitualmente construcciones antiguas. Con frecuencia es complicado obtener planos de calidad, pues los originales se pueden haber perdido, no han existido, o resultan imprecisos.

Con el desarrollo de las actuales tecnologías aparecen procedimientos de gran precisión, entre los que podemos señalar la estación total, la restitución fotográfica (1) y el escaneado láser 3D. Cabe esperar de estos métodos resultados más precisos que los obtenidos mediante los procedimientos tradicionales. El trabajo realizado mediante las nuevas técnicas, consistiría en que a partir del edificio y mediante los procesos de escaneado y manipulado de los resultados se realicen los planos de plantas y alzados del edificio.

No obstante, la arquitectura cuenta con propiedades y particularidades que en ocasiones dificultan la utilización de estas nuevas metodologías. En las edificaciones que alberguen grandes espacios diáfanos con superficies lisas, será muy apropiada la utilización de métodos como el escáner láser 3D. Sin embargo, cuando aparezcan espacios muy compartimentados, se requerirá que en cada estancia se realicen varios barridos, que posteriormente tendrán que ser unidos entre sí y cuyo resultado deberá enlazarse con el de los demás espacios. Aun admitiendo la gran exactitud de los resultados iniciales, es posible que los diversos procesos de montaje generen ciertos márgenes de error. Por este motivo queremos realizar un estudio comparativo en el que el proceso sea inverso: partir de un levantamiento y compararlo con los datos obtenidos de la nube de puntos.

La base del estudio será un edificio existente, con la particularidad de que se parte con documentación gráfica reciente, elaborada mediante métodos tradicionales, es decir, toma de datos de campo, mediante croquis en los que se anotan las medidas reales precisas para proceder al levantamiento del edificio.

Paralelamente se toma, como base de trabajo, un escaneado láser 3D realizado con anterioridad a la redacción del estudio, cedido por el Grupo de Investigación IGPU de la Universidad de Extremadura (2). Debido a que se trata de una actuación experimental, solo se escaneó parte del edificio, por lo que no se tienen datos globales del conjunto edificatorio.

A partir de esos dos soportes documentales previos, se elabora la base gráfica para su análisis comparativo. Por un lado, los planos obtenidos mediante métodos directos se trasladan a soporte vectorial informático, sobre los que se tomarán medidas mediante programas de dibujo asistido. Por otro lado, sobre los planos obtenidos a partir del escaneado láser existente, con soporte informático, también se tomarán medidas mediante programas de dibujo asistido. Las distancias que se comparen serán cotejadas con la realidad física del edificio. Para ello, los elementos de referencia serán claros de manera que no exista posibilidad de error en su localización: aristas definidas a cota constante.

Se analizan los resultados en dos planos característicos, correspondientes a zonas en las que no se tienen que realizar ajustes de conexiones entre los diversos escaneados; tampoco se tienen en cuenta los resultados correspondientes a los espacios muy compartimentados.

\subsection{Edificio objeto del levantamiento. Santuario de la Virgen de la Montaña}

El edificio está situado en la Sierra del Portanchito en la cima de un macizo rocoso de cuarcitas, al este de la ciudad de Cáceres y a una distancia aproximada del centro urbano de $4 \mathrm{Km}$. Se encuentra a una cota de 590 metros sobre el nivel del mar. El acceso se realiza a través de una carretera que facilita el tránsito rodado a lo largo de un itinerario con pronunciada pendiente y zonas con un trazado muy sinuoso (Figura 1 y Figura 2). Se tienen datos de su inicio a principios del siglo XVII. Está catalogado como espacio singular en el vigente planeamiento urbano.

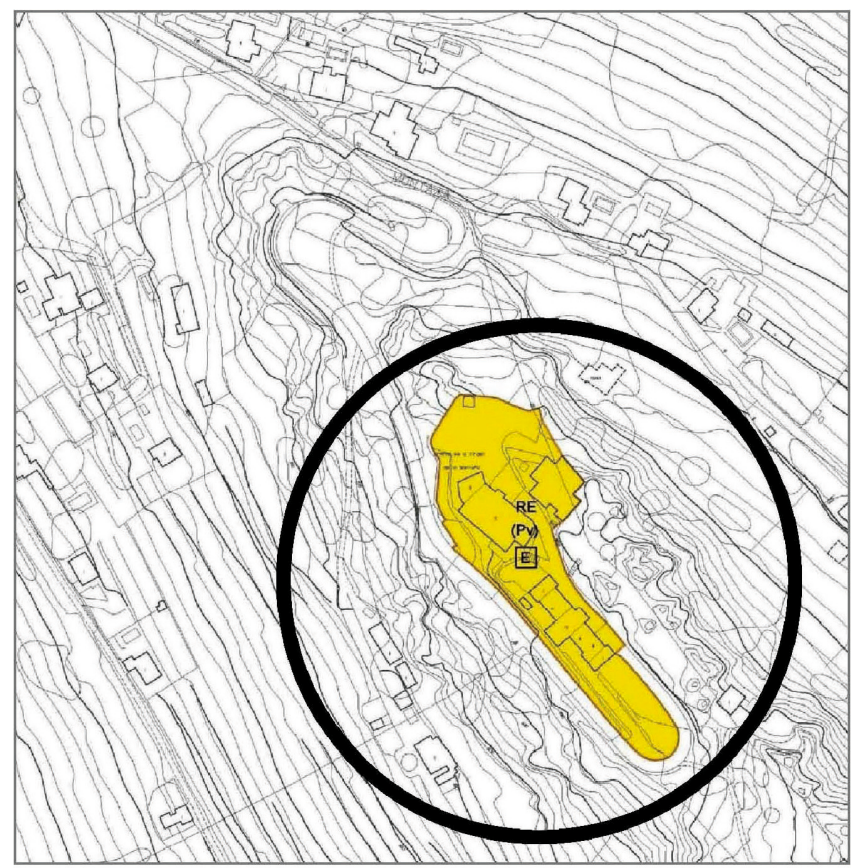

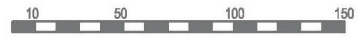

Figura 1. Plano de emplazamiento del conjunto edificado del Santuario de la Montaña en lo alto de la Sierra de la Mosca. (PROINTEC; (2010), Plan General de Ordenación Urbana de Cáceres. Ayuntamiento de Cáceres, Cáceres).

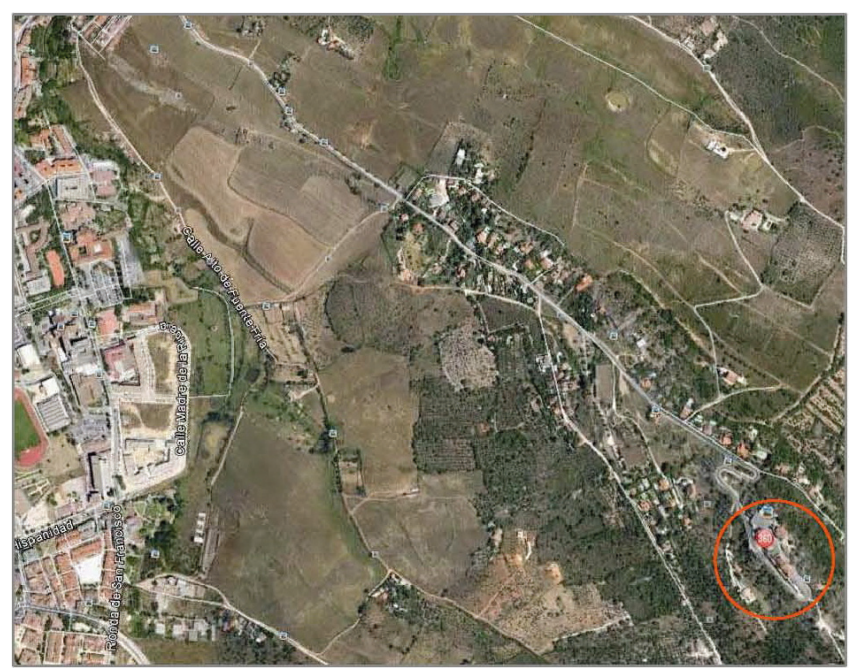

Figura 2. Situación del edificio al sureste de la ciudad de Cáceres en imagen aérea. (Google Maps). 
Según la tradición cristiana local, la Virgen se apareció a un pastor en una gruta excavada en la piedra. A partir de este hecho se realiza una capilla y, a continuación, diversas ampliaciones de distintos tamaños y tipologías, hasta dar con la actual edificación, dedicada al culto a la Virgen de la Montaña, patrona de la ciudad, que será el centro de nuestro trabajo.

El estudio consiste en la comparación entre los planos obtenidos por métodos tradicionales y los obtenidos a partir de una porción de puntos comprendida entre dos planos paralelos de la nube de puntos proporcionada por el escaneo realizado con el láser 3D.

Habitualmente se utilizan dos técnicas para trazar planos a partir de la nube de puntos. Una de ellas consiste en construir una malla a partir de los puntos y seccionarla obteniendo un conjunto de puntos coplanarios, que se unen en trazos conformando planos. La otra selecciona una porción (o rebanada) de puntos comprendidos entre dos planos paralelos separados una determinada distancia, a partir de los cuales se traza un dibujo en dos dimensiones. En este trabajo se utiliza esta segunda técnica.

Los planos a comparar serán dos: la planta de la capilla principal, seccionada a 1,20 metros respecto al suelo y una sección vertical longitudinal realizada por el centro de la capilla (Figura 3).

En esos planos de comparación se obtendrán las secciones, dibujadas en soporte informático a partir de la selección obtenida de la nube de puntos y se superpondrán con el trazado vectorial de los planos existentes, para poder comparar los resultados.

Posteriormente se procederá a tomar nuevas medidas en el modelo, es decir, en el propio edificio, en los mismos lugares, comparando los resultados y analizando su adecuación a la realidad.

\section{PARTE EXPERIMENTAL}

\subsection{Datos Previos}

El material con el que se cuenta consta de dos bloques de datos realizados con distintas tecnologías: planos de proyecto con medición directa y nubes de puntos procedente de un escaneado láser.

Por una parte, como parte de la realización del proyecto de rehabilitación del santuario, se obtiene la documentación gráfica correspondiente al levantamiento arquitectónico del estado previo del edificio. Comprende los planos que fueron visados por el Colegio Oficial de Arquitectos de Extremadura con fecha 27 de julio de 2011, que se tomará como fecha de referencia, aunque fueron realizados en marzo de 2010.

El resultado del levantamiento conlleva una serie de consideraciones previas que inciden cualitativamente en el resultado: su finalidad, los condicionantes del encargo y el plazo de ejecución. En primer lugar, el enfoque del trabajo será distinto si la finalidad del levantamiento es una toma de datos para documentar un bien patrimonial, acometer una rehabilitación o realizar una valoración económica. En el presente caso el levantamiento se realiza para ejecutar una rehabilitación, de manera que en él se refleja gráficamente su forma, omitiendo características constructivas o tipología de materiales. En segundo lugar, no existe un encargo específico de levantamiento de terceras personas, sino que se realiza para obtener una herramienta previa, de manera que pueda ser utilizada por el propio redactor del proyecto de rehabilitación. Por último, el tiempo del que se disponía se tuvo que repartir entre la realización del levantamiento y la redacción del propio proyecto.

El segundo bloque de material disponible para la comparación consiste en la toma de datos con láser escáner realizada como trabajo académico, sin condicionantes de encargo profesional, ni de plazos ni de finalidad precisa inmediata, realizada a lo largo del año 2011.

\subsubsection{Planos de levantamiento de proyecto}

Una vez tenidas en cuenta las consideraciones anteriores, se procede al levantamiento del edificio por métodos tradicionales, consistente en la realización de unos croquis previos y una posterior toma de cotas.

La toma de medidas se realiza mediante varios métodos: alineación referenciada, descomposición poligonal por triangulaciones, radiación, distancias parciales, acumuladas y totales y realización de croquis de detalle en tipos de huecos y elementos singulares.

Las herramientas de medida utilizadas han sido: flexómetro de $7 \mathrm{~m}$ de longitud y ancho $22 \mathrm{~mm}$, flexómetro de $3 \mathrm{~m}$ de longitud y ancho $15 \mathrm{~mm}$, cinta métrica de $50 \mathrm{~m}$ (Tarima Symrom-S), distanciómetro Láser (Leica Disto ${ }^{\mathrm{TM}}$ Classic).

\subsubsection{Escaneado Láser 3D}

El segundo bloque de datos consiste en un escaneado láser que nos proporciona una nube de puntos de la parte del edificio que vamos a analizar (Figura 4 ).

El número de estaciones utilizadas se puede advertir en la Figura 5: no se cuenta con un escaneado que comprenda la totalidad del mismo. No obstante, tal y como se indicó en la introducción, se pretende hacer un análisis delimitado en dos apartados muy específicos: la planta de la capilla principal y una sección transversal de la misma. En estos espacios sí se cuenta con suficiente información como para obtener una base de datos suficiente para realizar los planos de planta y sección.

El equipo con el que se ha realizado la toma de datos es una plataforma Leica Scan Station C10. Las especificaciones del equipo (3) son, en cuanto a rendimiento del sistema, en la precisión de medida aislada: posición $6 \mathrm{~mm}$ y distancia $4 \mathrm{~mm}$, ángulo (horizontal/vertical) $60 \mu \mathrm{rad} / 60 \mu \mathrm{rad}(12$ " / 12"); en la adquisición de objeto: $2 \mathrm{~mm}$ de desviación estándar. En lo referente al sistema de escaneo láser: sistema eléctrico: pulsado, microchip de propiedad; clase de láser: $3 \mathrm{R}$ (IEC 60825-1); alcance: hasta $300 \mathrm{~m}$ a $90 \%$; $134 \mathrm{~m}$ a $18 \%$ albedo (alcance mínimo o,1 m); velocidad de escaneo: hasta 50.000 puntos/seg. Resolución de escaneo: tamaño de punto: de o-50 m: 4,5 mm, (basado en FWHH), 7 mm (basado en Gaussian); resolución: horizontal y vertical totalmente 
seleccionable; espaciado mínimo de $<1 \mathrm{~mm}$; en todo rango, capacidad de intervalo de punto único.

Con este material se realizan diez estacionamientos que han sido montados utilizando el método de «Resección de dianas» con un software capaz de mover la elevada cantidad de puntos que se genera en el escaneo. Esta secuencia de operaciones es habitual en estos trabajos (4).

En la Figura 5 se señala la ubicación de ocho estacionamientos del total de los diez realizados: los seis de la derecha son los utilizados para la zona de estudio, los dos de la izquierda se corresponden con la gruta, situada a un nivel inferior. Los otros dos que no aparecen en la figura se realizan desde el exterior del edificio a una distancia y elevación que no influyen en los datos que se utilizan para tomar las referencias de las medidas estudiadas.

Con estos datos y una vez realizado el proceso de montaje de los diez estacionamientos, se ha exportado el resultado a un fichero en formato .dxf que puede ser manipulado con cualquiera de los programas de dibujo asistido que existen en el mercado, una de cuyas vistas se puede observar en la Figura 4.

Los equipos informáticos convencionales no pueden mover de manera eficiente mucho más de 15.000 puntos, mientras que el resultado del proceso realizado cuenta con más de 400 millones de puntos. En la exportación se realiza una reducción del número de puntos, aumentando la distancia entre los puntos de la nube a $30 \mathrm{~cm}$ de modo aleatorio. Realizar esta simplificación supone que solo se utiliza el o,00375 \% de la información que ofrece el resultado del escaneado de forma aleatoria, incidiendo en la precisión del resultado. Esto es un aspecto relevante a tener en cuenta, aunque los resultados se consideran válidos tomados en su conjunto, evaluando los puntos críticos en su contexto.

\subsection{Comparación}

A continuación se expone el material objeto del análisis. En la Figura 3 se presenta la planta de la capilla: en ella podemos ver la totalidad de la distribución de la planta del santuario, aunque el espacio que se comparará será el de la capilla, señalada con un fondo diferenciado y el rótulo «zona objeto de estudio».

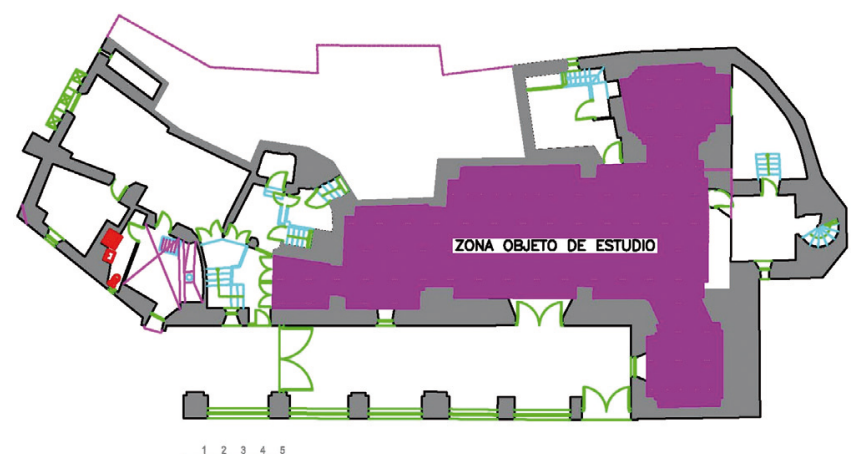

Figura 3. Zona objeto de estudio, en la nave principal del edificio. (Elaboración propia).

Otras imágenes recogen la nube de puntos orbitada (Figura 4) y la proyección en planta obtenida a partir de una selección de la nube de puntos con el perímetro interior de la planta de la capilla (Figura 5), de la que se han eliminado parte de los puntos exteriores: esta proyección servirá de base para la creación del dibujo de líneas con el que se comparará el trazado de la Figura 3.

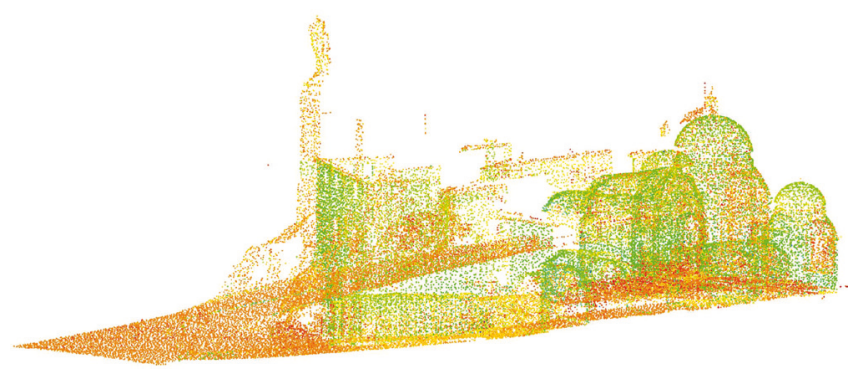

Figura 4. Nube de puntos orbitada. (Ingeniería Geomática y Patrimonio Urbano, IGPU. (2011). Toma de datos mediante láser escáner del Santuario de la Montaña. Universidad de Extremadura. Cáceres).

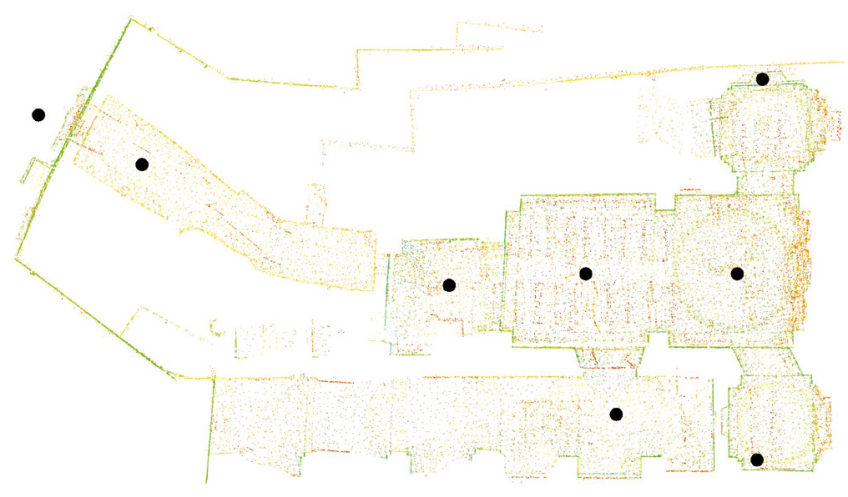

Figura 5. Planta de la capilla, cuya parte derecha se corresponde con la misma zona objeto de estudio de la Figura 3. Se señalan con puntos los 8 lugares de estacionamiento para las tomas con el láser escáner. . (Ingeniería Geomática y Patrimonio Urbano, IGPU. (2011). Toma de datos mediante láser escáner del Santuario de la Montaña. Universidad de Extremadura. Cáceres).

Siguiendo el procedimiento mencionado observamos que con la proyección obtenida a partir de la nube de puntos (Figura 5) se obtiene una base satisfactoria para poder dibujar el contorno del edificio y los espesores de sus muros exteriores (Figura 6).

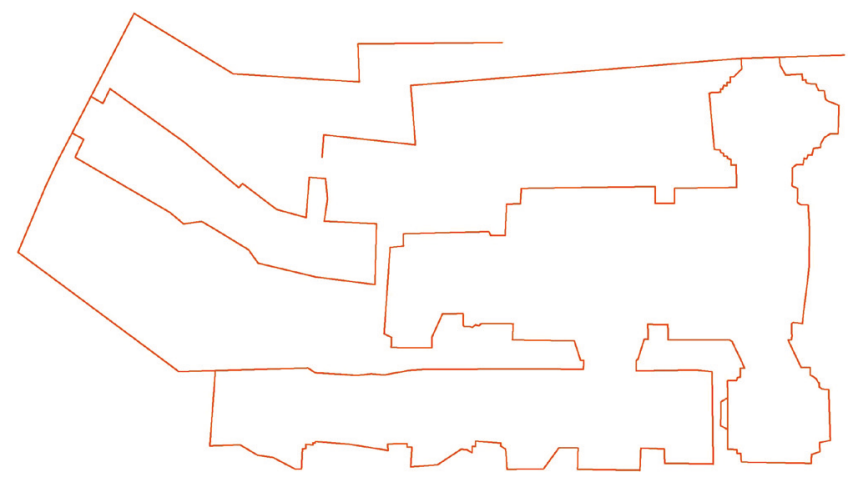

Figura 6. Trazado vectorial de la planta obtenido a partir de la nube de puntos. (Elaboración propia).

En el siguiente paso se superponen las dos plantas (Figura 7): se aprecian las diferencias existentes en el perímetro interior 
de la capilla, no existiendo diferencias substanciales entre las dos plantas (las que aparecen más llamativas se deben a la consideración de los huecos de fachada o al mobiliario, al variar la cota de referencia de la comparación), no obstante posteriormente se volverán a realizar tomas de cotas con respecto a puntos comunes de los elementos en los que existan diferencias en las dimensiones.

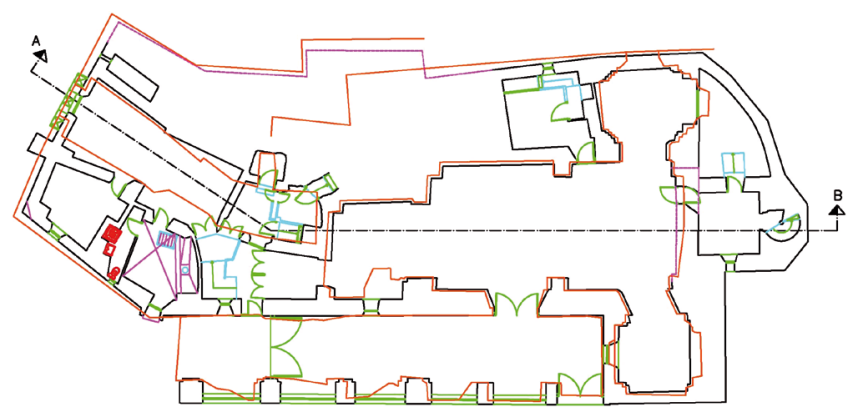

Figura 7. Superposición de los trazados vectoriales de las plantas de las Figuras 3 y 6 . (Elaboración propia).

Por otra parte, el análisis de la sección transversal es distinto al no haberse realizado un escaneo de la cubierta del edificio: sólo es válido para el interior. La toma exterior de datos resulta compleja de realizar por el entorno del edificio, de acusado relieve. Se podría contar con aparatos de medida suspendidos mediante globos o helicópteros, excediendo el objetivo del presente trabajo, para el que bastan las cotas interiores.

La situación de la sección longitudinal se señala en el plano de planta (Figura 7). La distancia entre los planos paralelos que limitan la porción de la nube de puntos para obtener la sección es de $30 \mathrm{~cm}$. Con este dato se puede advertir la precisión del resultado cuando la distancia de los puntos considerados en la nube de puntos es de $30 \mathrm{~cm}$, como se ha indicado al final del apartado 2.1.2.

De modo análogo a la planta, el plano de la sección de la capilla en soporte vectorial (Figura 8), se ha obtenido por métodos directos de medición. El perfil obtenido a partir de la nube de puntos (Figura 9) y el trazado vectorial a partir del mismo (Figura 10) completan la serie de trazados a cotejar. Podemos apreciar que, en comparación con la gran cantidad de puntos que contábamos para realizar la planta, los puntos que definen la sección de la capilla, a duras penas permiten adivinar el contorno de la sección, debido a que en la selección obtenida, la densidad de puntos es pequeña, tanto por la mayor distancia de los mismos desde la estación, como por la menor coincidencia entre las sucesivas nubes de puntos acumuladas.

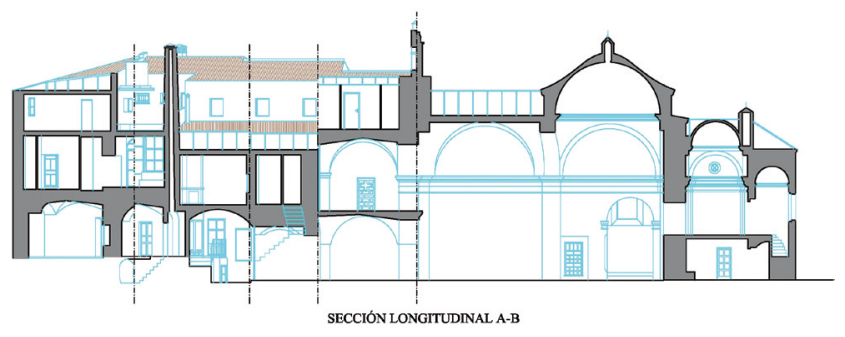

Figura 8. Soporte vectorial de la sección de la capilla. (Elaboración propia).

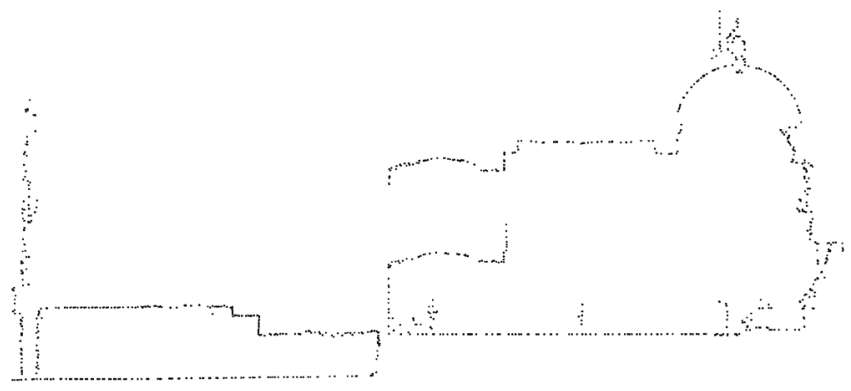

Figura 9. Nube de puntos correspondiente a la sección de la capilla. (Elaboración propia).

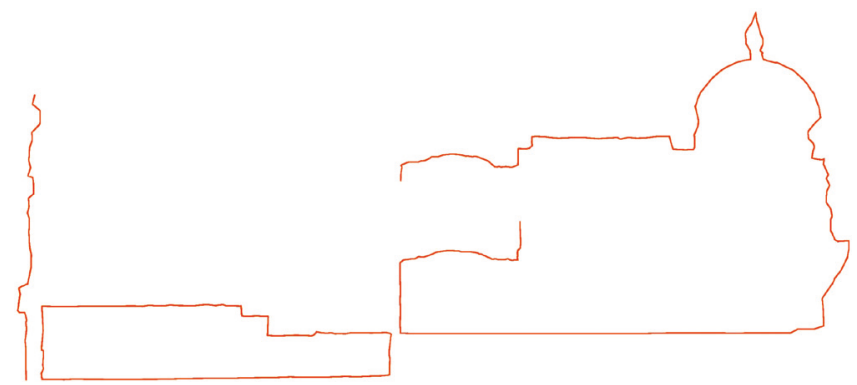

Figura 10. Trazado vectorial de la sección a partir de la nube de puntos. (Elaboración propia).

La superposición de las dos secciones (Figura 11) no ofrece un resultado con datos suficientes para realizar un análisis comparativo que sea representativo; no obstante tomaremos algunas distancias para ver la incidencia que tiene en las alturas, pues a menudo son datos que se toman de manera complicada mediante los procedimientos tradicionales.

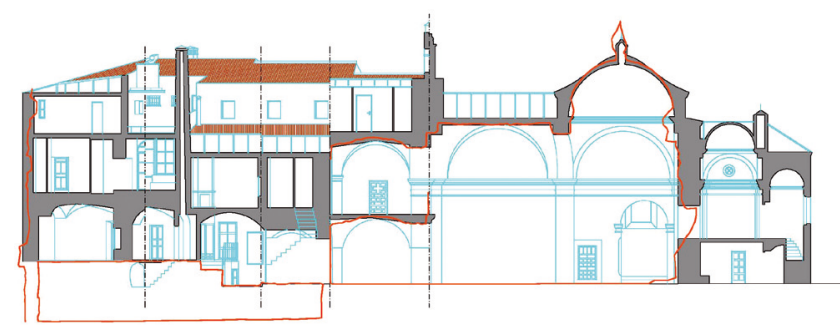

Figura 11. Superposición de los trazados vectoriales de las secciones de las Figuras 8 y 10. (Elaboración propia).

\section{RESULTADOS}

El propósito del estudio consiste por tanto en analizar el error existente en los dibujos realizados, de manera que obtengamos un orden de magnitud de los errores finales que aporta cada método. Para ello hemos comparado los planos trazados mediante métodos tradicionales con los obtenidos a partir de las porciones de la nube de puntos, manipuladas mediante ficheros de dibujo y, por tanto, menos densas.

Partimos de las superposiciones de trazados en planta (Figura 7) y en sección (Figura 11), para comparar medidas aisladas tomadas en los diversos soportes vectoriales y comprobadas posteriormente en el edificio, reflejando estas medidas en planta (Figura 12) y en sección (Figura 13).

En los dos casos los contornos son bastante parecidos: no obstante, hemos tomado medidas en las zonas en las que no 
existen concordancias. Se analiza la distancia entre elementos físicos que son representativos, es decir que tienen referencias fácilmente reconocibles tanto en los planos como en la realidad. Se evita, por tanto, tomar medidas superiores a $7 \mathrm{~m}$ que puedan provocar errores debido a la catenaria descrita por la cinta métrica en la medición directa. La altura a la que se toma la medida no influirá en el resultado al tratarse de elementos verticales sin desplomes, aunque se elige una altura uniforme de $1,20 \mathrm{~m}$, altura a la que se han realizado las secciones en planta. Ya se ha mencionado una posible divergencia debido a huecos de fachada o mobiliario, motivo por el cual se evitan esos lugares como puntos de comparación.

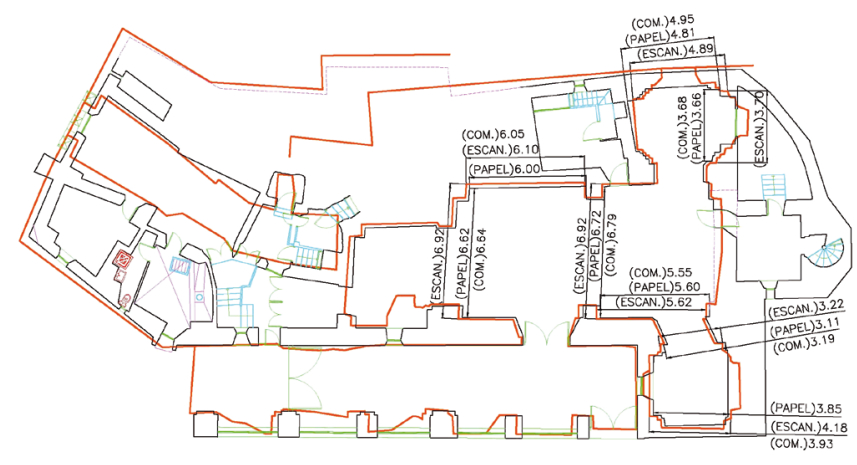

Figura 12. Superposición métrica de los trazados vectoriales de la planta (PAPEL. Fase 1. Medida directo trasladada a soporte vectorial: ESCAN. Fase 2. Toma de datos mediante escaneado láser trasladada a soporte vectorial; COM.

Fase 3. Comprobación puntual en el edificio por medida directa). (Elaboración propia).

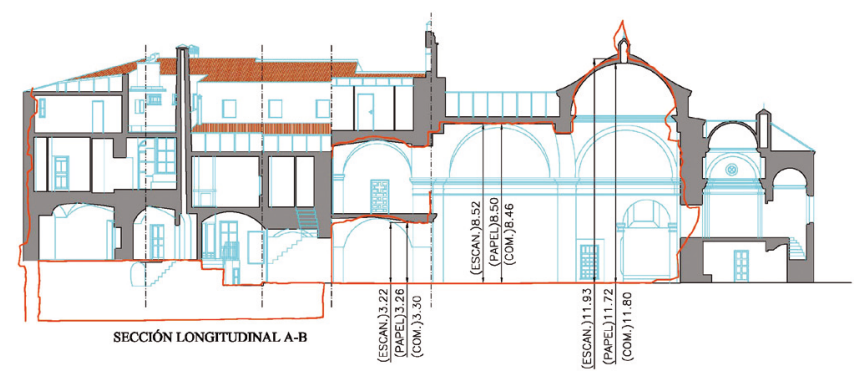

Figura 13. Superposición métrica de los trazados vectoriales de la sección (PAPEL. Fase 1. Medida directo trasladada a soporte vectorial: ESCAN. Fase 2. Toma de datos mediante escaneado láser trasladada a soporte vectorial; COM. Fase 3. Comprobación puntual en el edificio por medida directa). (Elaboración propia).
En los planos de sección vertical las comprobaciones de las alturas se han realizado únicamente mediante puntero láser. El proceso consiste en tomar primero la distancia en el dibujo en soporte vectorial correspondiente al plano de formato papel, a continuación se acota esa misma distancia en el dibujo en soporte vectorial obtenido a partir de la nube de puntos y por último se toma de nuevo referencia de la medida en el edificio, para comparar los tres datos. De esta manera vemos cual de los dos trazados es el más ajustado a la realidad.

Las medidas se toman siempre entre puntos correspondientes al mismo trazado. Como referencia de comparación, se realizan unas tablas (Tablas 1 y 2) en la que se transcriben los resultados de las medidas. En una primera fila, la comprobada en último lugar y, debajo, las resultantes de medir en el plano, que denominamos «papel», relativo al proyecto, y «escáner», procedente del láser. Se señalan las diferencias en metros de las mediciones comprobadas y las obtenidas de los dos tipos de planos. También se señala el porcentaje de error absoluto. Se puede apreciar que las mayores desviaciones respecto a la realidad comprobada se producen en los planos obtenidos a partir de la nube de puntos, con algunas puntas del 6,4 \% del valor real. Es interesante señalar que, si por una parte en los planos denominados «papel» el error suele ser por defecto, en las medidas tomadas en los planos de nube de puntos, el error es por exceso, lo que aumenta el arco de diferencia entre los dos modelos.

Las dimensiones tomadas en la sección (Tabla 2) aunque son más grandes, tienen menor error absoluto, con un porcentaje de error máximo del 2,4 \% en el caso del plano de la nube de puntos. Tenemos en cuenta que en el caso de las alturas es más complicado obtener mediciones correctas con métodos convencionales.

\section{DISCUSIÓN}

Con los resultados obtenidos tenemos una base para poder opinar sobre la efectividad de los métodos. Se señalan a continuación algunos aspectos de cada uno de ellos.

\subsection{Método tradicional}

No se pone en duda un procedimiento que se ha utilizado a lo largo de la historia de la arquitectura. No obstante en la actualidad se exige una gran precisión y efectividad visual. La precisión obtenida es aceptable y siempre se pueden obtener mejores resultados, dependiendo del grado de minuciosidad

Tabla 1. comparativa de medidas tomadas sobre la planta, a partir de la base vectorial impresa en PAPEL, nube de puntos procedente del láser ESCÄNER y medida COMPROBADA en el lugar. (Elaboración propia).

\begin{tabular}{|c|c|c|c|c|c|c|c|}
\hline & MEDIDA 1 & MEDIDA 2 & MEDIDA $3_{3}$ & MEDIDA 4 & MEDIDA 5 & MEDIDA 6 & MEDIDA 7 \\
\hline COMPROBADA & 6,64 & 6,05 & 6,79 & 5,55 & 3,19 & 3,93 & 4,95 \\
\hline PAPEL (m) & 6,62 & 6,00 & 6,72 & 5,6 & 3,11 & 3,82 & 4,81 \\
\hline $\operatorname{ESCANER}(\mathrm{m})$ & 6,92 & 6,1 & 6,92 & 5,62 & 3,22 & 4,18 & 4,89 \\
\hline \multicolumn{8}{|l|}{ ERROR } \\
\hline TOTAL PAPEL (m) & 0,02 & 0,05 & 0,07 & $-0,05$ & 0,08 & 0,11 & 0,14 \\
\hline$\%$ PAPEL & $0,3 \%$ & $0,8 \%$ & $1,0 \%$ & $-0,9 \%$ & $2,5 \%$ & $2,8 \%$ & $2,8 \%$ \\
\hline TOTAL ESCANER (m) & $-0,28$ & $-0,05$ & $-0,13$ & $-0,07$ & $-0,03$ & $-0,25$ & 0,06 \\
\hline \% ESCANER & $-4,2 \%$ & $-0,8 \%$ & $-1,9 \%$ & $-1,3 \%$ & $-0,9 \%$ & $-6,4 \%$ & $1,2 \%$ \\
\hline DIFERENCIA (m) & 0,3 & 0,1 & 0,2 & 0,02 & 0,11 & 0,36 & 0,08 \\
\hline
\end{tabular}


Tabla 2. comparativa de medidas tomadas sobre la sección, a partir de la base vectorial impresa en PAPEL, nube de puntos procedente del láser ESCÄNER y medida COMPROBADA en el lugar. (Elaboración propia).

\begin{tabular}{|l|c|c|c|}
\hline SECCION & MEDIDA 1 & MEDIDA 2 & MEDIDA 3 \\
\hline COMPROBADA & 3,3 & 8,46 & 11,80 \\
\hline PAPEL (m) & 3,26 & 8,50 & 11,72 \\
\hline ESCANER (m) & 3,22 & 8,52 & 11,93 \\
\hline & & & \\
\hline ERROR & 0,04 & $-0,04$ & 0,08 \\
\hline TOTAL PAPEL (m) & $1,2 \%$ & $-0,5 \%$ & $0,7 \%$ \\
\hline \% PAPEL & & & $-0,13$ \\
\hline TOTAL ESCANER (m) & 0,08 & $-0,06$ & $-1,1 \%$ \\
\hline \% ESCANER & $2,4 \%$ & $-0,7 \%$ & 0,21 \\
\hline
\end{tabular}

con el que se realice el trabajo. El propio proceso de levantamiento lleva inherente un estudio de la configuración formal y constructiva del edificio a dibujar, de manera que ha de ser realizado por técnicos especializados en edificación para que los resultados sean adecuados. En el ejemplo planteado tenemos un resultado bastante aceptable del grado de exactitud al que se puede llegar, teniendo en cuanta el valor de la inversión en personal que se realiza.

\subsection{Escaneado Láser}

Las nuevas tecnologías se abren camino en cualquier campo y el tamaño del patrimonio arquitectónico existente es descomunal, dando cabida a estos nuevos procesos. No obstante, precisa un gran despliegue de medios técnicos y humanos, con instrumentos de gran calidad y elevado coste, que además precisan un software poco difundido en la comunidad de técnicos que intervienen en el proceso del levantamiento no especializado. El resultado es, ante todo, espectacular desde un punto de vista visual. Para la obtención de planos arquitectónicos se requiere un proceso previo de manipulación de los primeros resultados que con frecuencia realizan técnicos especialistas, éstos crean unos archivos que a su vez vuelven a ser manipulados por otros técnicos de manera que el coste humano es mayor que el que se requiera en el método tradicional.

Los resultados se consideran aceptables, aunque el gran potencial de información aportado por el escaneado láser no puede ser aprovechado en toda su extensión con los actuales medios de realización de planos arquitectónicos. Por tanto se plantea una línea de investigación destinada a encontrar la manera de realizar la reducción del número de puntos mediante procedimientos (algoritmos matemáticos relacionados con la topología relativa de los puntos de la nube, por ejemplo) en los que el aspecto aleatorio se pueda minimizar, pudiéndose señalar zonas en las que se aporte mayor información y zonas en las que ésta se pueda desechar de manera voluntaria.

Por otra parte, entendemos que se debe profundizar en el análisis de programas que tiendan a confeccionar planos arquitectónicos a partir de la nube de puntos de manera que no se pierda precisión en la manipulación de los archivos. Los programas de dibujo no siempre están concebidos para mover el volumen de puntos que proporciona el escaneado y los programas que son capaces de moverlos no están ideados para realizar planos arquitectónicos.

La integración de esas dos ideas (reducción automática y equilibrada de la densidad de la nube de puntos y versatilidad del software empleado para su manipulación), podría significar el éxito de este proceso que de otro modo puede que no se generalice a corto plazo en un campo de trabajo tan extenso como es el del levantamiento de planimetría de edificios.

Otro factor a tener en cuenta en una comparación será un seguimiento de la autoría de la toma de datos con uno y otro método desde antes del inicio de los trabajos, de modo que se conozcan los objetivos precisos, para que las comparaciones sean más acertadas.

\section{REFERENCIAS}

(1) Santa Cruz-Astorqui, J. (2003). La fotogrametría digital en el levantamiento de planos de edificios. Informes de la Construcción, 55(488): 31-40, doi: http://dx.doi.org/doi:10.3989/ic.2003.v55.i488.539.

(2) Ingeniería Geomática y Patrimonio Urbano, IGPU. (2011). Toma de datos mediante láser escáner del Santuario de la Montaña. Cáceres: Universidad de Extremadura. (Inédito).

(3) Leica Geosystems AG.(2011). Leica ScanStation C-10: Manual de usuario. Heerbrugg, Suiza.

(4) Rubio, J., Baenar, J., Fernández, J.J., Finat, J. (2009). Nuevos métodos para viejas tecnologías: análisis y documentación de los materiales arqueológicos mediante la aplicación de sistemas Láser-scanner 3D. En I Congreso Internacional de Arqueología e Informática Gráfica, Patrimonio e Innovación. Sevilla. 